\title{
Suicide mitigation: time for a more realistic approach
}

One of the great myths of health politics is that risk can ultimately be eliminated if we have the right policies, procedures, and therapies. However, in areas where health professionals feel ill equipped to manage risk, those same expectations can be a powerful disincentive, discouraging the acknowledgement and assessment of risk.

When patients present with suicidal thoughts or following self harm, fear of accusation of suboptimal clinical expertise or even litigation if the patient goes on to complete a suicide may dissuade many clinicians from enquiring too deeply. This is in case they acknowledge a risk they will then be expected to manage, but which they do not believe they have the means to control. Thus, the pervasive expectation that risk must be controlled and preferably eliminated could paradoxically increase suicide risk rather than reduce it.

GPs appear willing to identify suicide risk in patients with a diagnosed mental illness. A study by Pearson et a/ investigated the frequency and nature of general practice consultations for 247 patients with a known mental illness, also in contact with specialist mental health services in the year before their suicide. In $91 \%$ of suicides the patient consulted their GP in the year before their death, half having their final GP consultation in the month before death and a sixth in the week before death.

Malone et al highlighted a lack of documentation of suicide risk by mental health professionals. ${ }^{2}$ Possible factors may include not appreciating that the patient was at risk from suicide or self harm, not asking the right questions, or not understanding what the patient wanted to convey. There may be difficulty with the process of identification, assessment, and response to suicide risk, particularly so in the case of young people visiting their GP before suicide. $^{3}$ Opportunities to intervene effectively may therefore be being missed.

A systematic review of suicide prevention studies in 2005 concluded that 'physician education' reduces suicide rates. ${ }^{4}$ The detection of suicidal intent, assessment of risk, and the possible prevention of suicide are in part dependent on a health professional's attitude to suicide prevention as being their responsibility or as an achievable clinical outcome. In a recent publication from The National Confidential Inquiry into Suicide and Homicide, 19\% of suicides $(n=1017)$ were considered preventable by the clinical teams seeing those patients. However, in $75 \%$ of suicides, clinicians believed that suicide risk factors could have been reduced. ${ }^{5}$ Of the suicides deemed to have been preventable, $12 \%$ ( $n=$ 122) were considered by the clinicians to be related to deficits in training.

It has been suggested that more robust training in suicide awareness in primary care could reduce morbidity and mortality. ${ }^{6}$ However, there are organisational and attitudinal issues that may be equally, or more, important for educators to consider than the selection of educational methods. These include making the training clinically relevant to GP consultations. ${ }^{7}$

Applied Suicide Intervention Skills Training (ASIST) within community-based suicide prevention strategies has already been adopted in many countries, including Canada, the US, Australia, Scotland, and Ireland. In Wales, the Welsh Assembly Government has decided that there is also a need for shorter, more clinically-based training to enable busy GPs to receive training in suicide and self-harm awareness and improve their skills in these areas. ${ }^{8}$ Accordingly, a training package, 'Connecting with People', is being developed and tailored in length and content to increase the relevance and impact for primary care. ${ }^{9}$ It will complement the 2-day ASIST training and encourage GPs to attend the full programme. It will be evaluated by the National Public Health Service in Wales.

To dispel the myth that suicide risk can somehow be controlled and eliminated by the use of tools and a 'risk management system', we believe that the concept of risk mitigation, rather than risk management, should be the cornerstone of any community suicide prevention strategy. This will require challenging the culture of 'risk management' with a more realistic approach based on risk mitigation, and improving the ways in which suicide risk is understood, assessed, and responded to in all sectors.

Risk mitigation is not a soft option to avoid responding to identified risks, but an active process involving collaboration of the clinical team with the patient and their carers. Some risk factors, such as demographics or life events, cannot be reduced, but we propose that they could be mitigated against and potentially 'neutralised'. For example, old age is not in itself a reason for suicide. However, some of the factors accompanying old age, such as social isolation, bereavement, loss of role, and ill health, are known risk factors. Mitigating those factors that are open to influence within primary care, and agreeing a collaborative, simple suicide mitigation plan could reduce suicide risk. In addition to the therapeutic relationship, this should be underpinned by enabling the patient to resist acting on their suicidal thoughts.

The risk mitigation approach emphasises the degree of ambivalence felt by many suicidal individuals and challenges the common perception that it is not possible for someone without specialist psychiatric knowledge and training to help a suicidal individual resist acting on their suicidal thoughts. Increasing hopefulness, resilience, and reasons for living have been shown to reduce suicide rates, ${ }^{10}$ and GPs can play a crucial role in challenging ideas of hopelessness.

Suicide and self-harm reduction can be viewed in the same way as preventative measures for any other medical condition with multifactorial risk factors, such as heart disease, cancer, and obesity. Knox et al state that 50 years ago treatment for heart disease meant acute hospital care for myocardial infarction. A study reported that GPs surveyed recognise the importance of a public health approach and actively treat cardiovascular risk factors, and they ask why this is not the case with suicide prevention. ${ }^{11}$ One might speculate that it is the active 
event that is required for a suicide to occur in contrast to the rather more passive event of myocardial infarction that may lead some people to view suicide attempts differently from cardiac disease.

We believe that any suicide prevention strategy should be community based. The use of a risk-assessment form as a 'tick box' exercise to show that the correct risk management procedure has been followed is unlikely to prevent suicides. However, to have the greatest chance of mitigating against an identified risk of suicide, we would advocate an empathic therapeutic relationship in addition to a diligent identification and discussion of the particular risk factors in a person's background and skilful clinical assessment including mental state examination. ${ }^{12,13}$

Instead of the unrealistic clinical goal of a 'cure' for some individuals' suicidal intent, the question practitioners could be asking is: 'What can be done to prevent this person harming themselves today, this week, this month?'. In all fields of medicine, prolonging life and improving the quality of life, rather than cure, is accepted by professionals, patients, politicians, and society at large as an appropriate clinical objective and outcome for many patients. GPs never know in which one of their patients their intervention will have saved a life: suicidal intent should be viewed in the same way. This is especially so in the case of heart disease, with statins having a 'number needed to treat' of $95 .{ }^{14}$ This intervention comes at a price (financial, and with adverse side effects), yet GPs will prescribe this knowing that in 94 cases it will probably be ineffective and possibly even cause harm. There is no evidence that a therapeutic relationship and identifying risk and protective factors is harmful, on the contrary it has shown to reduce suicides. ${ }^{10,15}$

In addition to suicide awareness and skills training, this new approach will require enhancing shared decision making with patients and their carers, the development of a common language and understanding between different sectors, improved availability of psychosocial interventions, ${ }^{16}$ and improved consistency of response at all points of contact with suicidal individuals.

\section{Alys Cole-King,}

Liaison psychiatrist, Betsi Cadwaladr University Health Board, North Wales.

\section{Peter Lepping,}

Consultant psychiatrist, Wrexham Academic Department of Mental Health, Betsi Cadwaladr University Health Board, Wrexham.

\section{OnIne version}

This is the full-length editorial of an abridged version published in print.

\section{Provenance}

Freely submitted; peer reviewed.

\section{Acknowledgements}

The authors wish to thank Dr Huw Lloyd, a GP colleague, for his help and encouragement with the ideas presented in this paper.

\section{REFERENCES}

1. Pearson A, Saini P, Da Cruz D, et al. Primary care contact prior to suicide in individuals with mental illness. Br J Gen Pract 2009; 59(568): 825-832.

2. Malone KM, Szanto K, Corbitt EM, Mann JJ. Clinical assessment versus research methods in the assessment of suicidal behaviour. Am J Psychiatry 1995; 152(11): 1601-1607.

3. Appleby L, Amos T, Doyle U, et al. General practitioners and young suicides: a preventive role for

\section{Box 1. Risk factors for suicide mitigation.}

\begin{tabular}{l} 
Demographic and \\
social factors \\
\hline Demographic: \\
age and sex; marital status; \\
employment status; certain \\
professions (farmers, vets, \\
doctors, and dentists); economic \\
class (high and low); minority \\
groups. \\
Life events: \\
especially if involving loss and \\
repeated or chronic; childhood \\
adversity, especially abuse; \\
recent bereavement; \\
relationship instability; redundancy; \\
loss of privilege. \\
Lack of social capital: \\
social isolation and living alone; \\
lack social support; \\
institutionalisation, \\
for example, prison.
\end{tabular}

Access to lethal means: firearms; suicide 'hot spots'.
Personal background

Family history:

suicide and mental illness, particularly alcoholism and bipolar affective disorder.

Personality disorder: personality traits: - impulsivity

- aggression

- lability of mood.

Substance misuse: precipitated by loss of interpersonal relationships. Especially if: - high level of dependency - long history of drinking

- depressed mood

- poor physical health

- poor work record in last 4 years (peak age 40-60 years).

Poor problem solving skills: low IQ; poor coping skills.
Clinical factors in history

\section{Mental illness:}

repeated relapses; recent scharge from psyatric unit; recent relapse in mental illness; self neglect; agitation; depressive symptoms, especially anhedonia and insomnia; severe anxiety and panic; psychotic symptoms; chronic schizophrenia, recurrent depression.

\section{Previous self harm:}

especially if previous high suicide intent attempt, superficial cutting, recent increasing intent if repeated self harm.

Chronic medical illness: beware if the medical condition is accompanied by pain and functional disability; chronic pain, cancer; cardiovascular disease; dialysis; GI disease; male patients with GU disease; neurological illness: epilepsy (especially early onset), MS, dementia, post-cerebral trauma.

Medication:

both prescribed and over the counter; (consider weekly scripts).
Mental State Examination (particularly suicidal thoughts)

\section{Hopelessness:}

difficulty seeing any future; particularly

worrying if only able to see 1-2 hours

future; 'nothing to live for'; negative

thoughts, helplessness, guilt,

'I'm a burden'.

Suicidal ideas and plans: especially if recent worsening and associated with distress; suicide plans/preparations, e.g. will, note, internet, lethality of chosen method, and possible rescue/treatment. Beware, as proposed method can easily change from a low-lethality method to a high-lethality method.

Psychotic symptoms:

distressing psychotic phenomena persecutory delusions, nihilistic delusions; command hallucinations perceived as omnipotent.

\section{Cognitive functioning}

delirium, low IQ.

Insight:

previous high pre-morbid functioning and fear of deterioration; early stage of illness.

GI = gastrointestinal. $G U=$ genitourinary. $M S=$ multiple sclerosis. 
primary care. Br J Psychiatry 1996; 168(3): 330-333.

4. Mann JJ, Apter A, Bertolote J, et al. Suicide prevention strategies: a systematic review. JAMA 2005; 294(16): 2064-2074.

5. Appleby L, Shaw J, Kapur NN, et al. Avoidable deaths: five year report of the National Confidential Inquiry into Suicide and Homicide by People with Mental Illness. Manchester: The University of Manchester, 2006.

6. Sudak D, Roy A, Sudack H, et al. Deficiencies in suicide training in primary care specialties: a survey of training directors. Acad Psychiatry 2007; 31(5): 345-349.

7. Hodges B, Inch C, Silver I. Improving the psychiatric knowledge, skills, and attitudes of primary care physicians, 1950-2000: a review. Am J Psychiatry 2001; 158(10): 1579-1586.

8. Welsh Assembly Government. 'Talk to me': the national action plan to reduce suicide and self harm in Wales 2009-2014. Welsh Assembly Government, 2009.

9. Cole-King A, Slegg GP, Peake-Jones GC. Evaluation of suicide risk assessment and self-harm awareness training [abstract]. Royal College of Psychiatrists, Faculty of Liaison Psychiatry Annual Meeting, Prague, Czech Republic, 2009.

10. McLean J, Maxwell M, Platt S, et al. Risk and protective factors for suicide and suicidal behavior: a literature review. Edinburgh: The Scottish Government, 2008.

11. Knox KL, Conwell Y, Caine ED. If suicide is a public health problem, what are we doing to prevent it? Am J Public Health 2004; 94(1): 37-45.

12. Gliatto MF, Rai AK. Evaluation and treatment of patients with suicidal ideation. Am Fam Physician 1999; 59(6): 1500-1506.

13. Cole-King A, Lepping P, Peake-Jones G, et al. The ColeKing Mitigation Framework: evaluation of a framework for supporting the identification and response to suicide risk [abstract]. Welsh Division of the Royal College of Psychiatrists Annual Conference,
Ewloe, UK, 2009.

14. National Institute for Health and Clinical Excellence. Technology Appraisal 94: statins for the prevention of cardiovascular events, London: NICE, 2006. [section 4.1.7]

15. Collins S, Cutcliffe IS. Addressing hopelessness in people with suicidal ideation: building upon the therapeutic relationship utilizing a cognitive behavioural approach. J Psychiatr Mental Health Nurs 2003; 10(2): 175-185.

16. Lepping P, Woodworth B, Roberts L, Turner J. Increasing psychosocial assessment by introducing self-harm pathway. Psychiatric Bulletin 2006; 30: $69-172$

DOI: 10.3399/bjgp10X482022

\section{ADDRESS FOR CORRESPONDENCE}

\section{Alys Cole-King}

Betsi Cadwaladr University Health Board, Department of Liaison Psychiatry, Maelor Hospital, Croesnewydd Road, Wrexham,

LL13 7TD.

E-mail: ALYS.COLE-KING@wales.nhs.uk 